\title{
Topological Black Holes in Weyl Conformal Gravity
}

\author{
Dietmar Klemm* \\ Dipartimento di Fisica, Università di Trento, Italia
}

\begin{abstract}
We present a class of exact solutions of Weyl conformal gravity, which have an interpretation as topological black holes. Solutions with negative, zero or positive scalar curvature at infinity are found, the former generalizing the wellknown topological black holes in anti-de Sitter gravity. The rather delicate question of thermodynamic properties of such objects in Weyl conformal gravity is discussed; suggesting that the thermodynamics of the found solutions should be treated within the framework of gravity as an induced phenomenon, in the spirit of Sakharov's work.
\end{abstract}

04.20.-q, 04.20.Gz, 04.70.Bw

*email: klemm@science.unitn.it 


\section{INTRODUCTION}

Topological black holes [1] 6] represent an interesting domain of general relativity. They are expected to occur naturally in any future theory of quantum gravity involving topological transition processes, and they may provide further ground to test string theory ideas in black hole physics [7]. A necessary condition for their existence seems to be the presence of a negative cosmological constant, i. e. they occur in anti-de Sitter (AdS) gravity. Indeed, Hawking's theorem [8] states that under the assumption of asymptotic flatness and positivity of matter-energy, the event horizon of a black hole is always spherical.

Now in the action of Weyl conformal gravity there is no room for a cosmological constant, as this would introduce a length scale and hence break conformal invariance. This raises the question if objects like topological black holes can nevertheless exist in such a theory

of gravitation. (Note in this context that recently there have been found exact solutions of other higher derivative theories, which represent black holes in an AdS background [9]. In this work, the cosmological constant $\Lambda$ arises as a product of the integration procedure, and is not taken as an ingredient of the action. However, the field equations are solved only for special values of $\Lambda$ ).

In this letter we want to investigate if Weyl conformal gravity admits solutions representing topological black holes. We do not discuss the advantages or drawbacks of this alternative theory of gravitation, as there is a vast list of literature dedicated to this subject. In any case, it seems interesting to consider black holes in Weyl gravity, because the requirement of conformal invariance at the classical level leads to a renormalizable gauge theory of gravity, which permits a consistent picture of black hole evaporation [10].

The remainder of this paper is organized as follows:

In section II we review the gravitational action, the field equations and the most general spherically symmetric solution of Weyl conformal gravity.

In section III exact solutions having an interpretation as black holes with non-trivial topology

are derived. As a special case we will recover the well-known topological black holes in AdS gravity, but we also find other cases which, in contrast to the former, have non-negative scalar curvature at infinity.

In section [V] we discuss the black holes' thermodynamic properties, which will result to be a rather ticklish concern, e. g. applying Wald's definition of thermodynamic variables for an arbitrary, diffeomorphism-invariant Lagrangian [12], seems to yield only trivial results in the case of black holes in Weyl conformal gravity. We therefore suggest a possible treatment of the thermodynamics of the found solutions by the idea of gravity as an induced phenomenon 11].

Finally, our results are summarized and discussed in section $\square$.

\section{WEYL CONFORMAL GRAVITY}

The conformally invariant action under consideration is

$$
I_{W}=\alpha \int d^{4} x \sqrt{-g} C_{\mu \nu \rho \lambda} C^{\mu \nu \rho \lambda}
$$




$$
=2 \alpha \int d^{4} x \sqrt{-g}\left[R_{\mu \nu} R^{\mu \nu}-\frac{1}{3}\left(R_{\mu}^{\mu}\right)^{2}\right]
$$

where $C_{\mu \nu \rho \lambda}$ is the Weyl tensor and $\alpha$ is a dimensionless parameter, which is usually chosen to be positive, in order to have a positive definite euclidean action and an acceptable newtonian limit.

The vacuum field equations following from (11) read [13 15]

$$
W_{\mu \nu}=W_{\mu \nu}^{(2)}-\frac{1}{3} W_{\mu \nu}^{(1)}=0
$$

where $W_{\mu \nu}^{(1)}$ and $W_{\mu \nu}^{(2)}$ are given by

$$
\begin{aligned}
& W_{\mu \nu}^{(1)}=2 g_{\mu \nu} \nabla_{\beta} \nabla^{\beta} R_{\alpha}^{\alpha}-2 \nabla_{\nu} \nabla_{\mu} R_{\alpha}^{\alpha}-2 R_{\alpha}^{\alpha} R_{\mu \nu}+\frac{1}{2} g_{\mu \nu}\left(R_{\alpha}^{\alpha}\right)^{2}, \\
& W_{\mu \nu}^{(2)}=\frac{1}{2} g_{\mu \nu} \nabla_{\beta} \nabla^{\beta} R_{\alpha}^{\alpha}+\nabla_{\beta} \nabla^{\beta} R_{\mu \nu}-\nabla_{\beta} \nabla_{\nu} R_{\mu}^{\beta}-\nabla_{\beta} \nabla_{\mu} R_{\nu}^{\beta}-2 R_{\mu \beta} R_{\nu}^{\beta}+\frac{1}{2} g_{\mu \nu} R_{\alpha \beta} R^{\alpha \beta} .
\end{aligned}
$$

As $W_{\mu \nu}^{(1)}$ and $W_{\mu \nu}^{(2)}$ vanish identically for $R_{\mu \nu}=\Lambda g_{\mu \nu}$, this implies that every Einstein space also satisfies the vacuum field equations of Weyl conformal gravity. As the topological black holes discussed in [1-6] are Einstein spaces (if uncharged), they also fulfill (2). However, inspecting the most general static, spherically symmetric solution of (2) (see below) derived in [14, we expect the field equations of Weyl conformal gravity to admit also a richer class of topological black hole solutions, which will not consist of Einstein spaces alone. As we will see in section 【II, this is indeed the case.

The general static, spherically symmetric line element resolving (2) reads [14]

$$
d s^{2}=-V(\rho) d \tau^{2}+V(\rho)^{-1} d \rho^{2}+\rho^{2}\left(d \Theta^{2}+\sin ^{2} \Theta d \Phi^{2}\right),
$$

with $V(\rho)$ given by

$$
V(\rho)=1-\frac{\beta(2-3 \beta \gamma)}{\rho}-3 \beta \gamma+\gamma \rho-k \rho^{2},
$$

$\beta, \gamma$ and $k$ being constants. (Of course multiplying (4) by a conformal factor depending only on $\rho$, one again obtains a static, spherically symmetric solution). One notes that for $\gamma=0$, (5) yields the Schwarzschild-, Schwarzschild-(anti-)de Sitter-, and (anti-)de Sitter spacetimes, depending on the choice of the parameters, but for $\gamma \neq 0$ there arises an additional potential linear in $\rho$.

\section{TOPOLOGICAL BLACK HOLE SOLUTIONS}

To obtain topological black holes, we analytically continue (四) according to

$$
\tau=i t, \quad \rho=i r, \quad \Theta=i \theta, \quad \Phi=\phi, \quad \beta=-i b, \quad \gamma=i c,
$$

which yields 


$$
d s^{2}=-V(r) d t^{2}+V(r)^{-1} d r^{2}+r^{2}\left(d \theta^{2}+\sinh ^{2} \theta d \phi^{2}\right),
$$

where $V(r)$ now is given by

$$
V(r)=-1-\frac{b(2-3 b c)}{r}+3 b c+c r-k r^{2} .
$$

For suitable values of the parameters, (7) describes a black hole spacetime. The metric induced on the spacelike surfaces of constant $r$ and $t$, in particular on the event horizon, is

$$
d \sigma^{2}=r^{2}\left(d \theta^{2}+\sinh ^{2} \theta d \phi^{2}\right)
$$

This is the standard metric of hyperbolic two-space $\mathrm{H}^{2}$, which has constant negative curvature and, of course, is not compact. We now compactify the $(\theta, \phi)$-sector by acting with an appropriate discrete subgroup $G$ of the isometry group $\mathrm{SO}(2,1)$ of $\mathrm{H}^{2}$, i. e. we consider the quotient space $\mathrm{H}^{2} / G$, which is compact. If we require it to be orientable, it becomes a Riemann surface $S_{g}$ of genus $g>1$, and the topology of the four-dimensional manifold is $\mathbb{R}^{2} \times S_{g}$. (For an exhaustive description of the compactification procedure see e. g. [16])

Setting $c=0$ and $k=\Lambda / 3<0, \Lambda$ being the cosmological constant, one recovers the wellstudied uncharged, static topological black hole solutions in AdS gravity. Note however that now we have further possibilities to construct black holes with non-trivial topology. E. g. we may set $k=0$, yielding a solution which is not asymptotically AdS. For $c>0$ and $-1 \leq 3 b c<2$ it has a black hole interpretation, $3 b c=-1$ yielding an extreme black hole. The scalar curvature for $k=0$ is given by

$$
R=-\frac{6 c}{r}\left(1+\frac{b}{r}\right),
$$

hence we have a curvature singularity at $r=0 . R$ vanishes for $r \rightarrow \infty$. Note however that the manifold is not Ricci-flat at infinity, therefore it does not approach flat Minkowski space, and we have no contradiction to Hawking's theorem [8], because this makes essential use of asymptotic flatness. In the case $k=0$, the manifold is globally hyperbolic, in contrast to AdS black holes.

One further observes that (8) is equivalent to (5), which can easily be seen by setting

$$
c=\gamma, \quad b=-\beta+\frac{2}{3 \gamma} .
$$

Consequently the lapse functions for the spherical and the $g>1$ black hole are identical, a feature different from black holes in AdS gravity. (Note however, that the full metrics (田) and (7) clearly are not equivalent).

Now set e. g. $b=2 / 3 c-\eta$ and $k=\Lambda / 3>0$ in (8), giving in the limit $c \rightarrow 0$

$$
V(r)=1-\frac{2 \eta}{r}-\frac{\Lambda r^{2}}{3}
$$

This represents a black hole spacetime similar to the Schwarzschild-de Sitter solution, but now with non-trivial topology. The scalar curvature approaches $4 \Lambda$, as $r$ goes to infinity. It 
is important to stress that (12) is not a solution of Einstein's equations $R_{\mu \nu}=\Lambda g_{\mu \nu}$, as in this case a lapse function

$$
V(r)=C-\frac{2 \beta}{r}-\frac{\Lambda r^{2}}{3}
$$

would only admit horizons whose curvatures have the same sign as the constant $C$, i. e. spherical ones for (12).

For $9 \beta^{2} \Lambda<1$ (12) has two zeroes $r_{-}<r_{+}, r_{-}$representing the black hole's event horizon, whereas $r_{+}$is a cosmological horizon. The occurence of topological black holes which are neither asymptotically flat nor AdS is also known in dilaton gravity 17.

In order to obtain a toroidal black hole spacetime, one can perform another analytical continuation of (4) by

$$
\tau=t \sqrt{d}, \quad \rho=\frac{r}{\sqrt{d}}, \quad \Theta=\theta \sqrt{d}, \quad \Phi=\phi, \quad \beta=\frac{b}{\sqrt{d}}, \quad \gamma=\frac{c}{\sqrt{d}} .
$$

Taking the limit $d \rightarrow 0$, we get

$$
d s^{2}=-V(r) d t^{2}+V(r)^{-1} d r^{2}+r^{2}\left(d \theta^{2}+\theta^{2} d \phi^{2}\right)
$$

with

$$
V(r)=\frac{3 b^{2} c}{r}-3 b c+c r-k r^{2}
$$

For suitable parameter values, (15) represents a black hole. The $(\theta, \phi)$-sector carries now the flat metric

$$
d \sigma^{2}=r^{2}\left(d \theta^{2}+\theta^{2} d \phi^{2}\right)
$$

Passing from polar to cartesian coordinates according to

$$
\begin{aligned}
& x=\theta \cos \phi, \\
& y=\theta \sin \phi,
\end{aligned}
$$

and identifying

$$
\begin{aligned}
& x \simeq x+n, \\
& y \simeq y+m,
\end{aligned}
$$

with $n, m \in Z$, we get a compact orientable surface with genus $g=1$, i. e. a torus, and the topology of our four-dimensional manifold becomes $\mathbb{R}^{2} \times S^{1} \times S^{1}$.

Setting $c=-2 \eta / L, b=\sqrt{L / 3}, k=\Lambda / 3<0$, yields in the limit $L \rightarrow \infty$

$$
V(r)=-\frac{2 \eta}{r}-\frac{\Lambda r^{2}}{3}
$$

which desrcibes for $\eta>0$ the uncharged static toroidal black hole known from AdS gravity. Note that, unlike the $g>1$ case, now for $k \geq 0$ one looses the black hole interpretation, as 
(16) does not have any real root for $k=0$, and only one real root for $k>0$, which is not a black hole event horizon.

Now one may doubt if the analytical continuations desribed above yield again solutions of (2); especially the limit procedure to obtain the toroidal case may appear somewhat doubtful. Therefore, following the derivation of the spherically symmetric case in [14], we make the ansatz

$$
d s^{2}=-f(\rho) d t^{2}+g(\rho) d \rho^{2}+\rho^{2} d^{2} \Omega
$$

for the static topological black hole spacetime, where $d^{2} \Omega$ is given by

$$
d^{2} \Omega=\left\{\begin{aligned}
d \theta^{2}+\theta^{2} d \phi^{2} & , \quad g=1 \\
d \theta^{2}+\sinh ^{2} \theta d \phi^{2} & , \quad g>1
\end{aligned}\right.
$$

An analogous calculation to [14 then yields exactly the solutions above, namely (8) resp. (16), confirming the validity of our analytical continuations.

\section{THERMODYNAMIC PROPERTIES}

Let us now discuss the thermodynamic properties of the black hole solutions found above. First we remark that in the derivation of the Hawking effect 18 the field equations do not enter, so a black hole will emit Hawking radiation at temperature $\kappa / 2 \pi$ in an arbitrary theory of gravitation ( $\kappa$ being the surface gravity). Therefore it makes sense to assign the temperature $T=\kappa / 2 \pi$ with $\kappa=V^{\prime}\left(r_{H}\right) / 2$ to our topological black holes found above. $\left(r_{H}\right.$ denotes the event horizon radial coordinate). As for the entropy, Wald showed [12,19] that in an arbitrary theory of gravity with diffeomorphism-invariant Lagrangian, one can define black hole entropy as the Noether charge associated with the local symmetry generated by the horizon Killing field. He further demonstrated the validity of a first law of thermodynamics under the assumption of asymptotic flatness. (See also 20 22 for black hole thermodynamics in a generalized theory of gravity). However the topological black holes in Weyl conformal gravity are not asymptotically flat, so the validity of a first law remains to be verified. The definition of the mass given in [12 is not applicable in the case of non asymptotic flatness; a suitable background subtraction has to be carried out. Yet, as we suspect the entropy to be a local quantity closely connected to horizon properties, it should be independent of the asymptotic behaviour. So we are led to apply the Noether charge definition given in [12] also to the topological black holes found above. As is shown in [20, the entropy $S$ is then given by

$$
S=-2 \pi \oint_{\Sigma} Y^{\mu \nu \rho \lambda} \hat{\epsilon}_{\mu \nu} \hat{\epsilon}_{\rho \lambda} \bar{\epsilon}
$$

where

$$
Y^{\mu \nu \rho \lambda}=\frac{\partial \tilde{L}}{\partial R_{\mu \nu \rho \lambda}}
$$


$\tilde{L}$ denoting the scalar Lagrangian density $\left(I=\int d^{4} x \sqrt{-g} \tilde{L}\right)$. $\hat{\epsilon}_{\mu \nu}$ is the binormal to the (arbitrary) cross-section $\Sigma$ of the horizon, and $\bar{\epsilon}$ the induced volume form on $\Sigma$. For the action (11), $Y^{\mu \nu \rho \lambda}$ reads

$$
\begin{aligned}
Y^{\mu \nu \rho \lambda}= & \alpha\left(g^{\mu \rho} R^{\nu \lambda}-g^{\nu \rho} R^{\mu \lambda}-g^{\mu \lambda} R^{\nu \rho}+g^{\nu \lambda} R^{\mu \rho}\right) \\
& -\frac{2 \alpha}{3} R_{\beta}^{\beta}\left(g^{\mu \rho} g^{\nu \lambda}-g^{\nu \rho} g^{\mu \lambda}\right) .
\end{aligned}
$$

Now consider e. g. the $g>1$ black hole (77) with lapse function (12) and $\Lambda=0$. Using the theorem of Gauss-Bonnet, the entropy (23) is then easily calculated, and reads

$$
S=\frac{256 \pi^{2} \alpha}{3}(g-1) .
$$

Hence we obtained a result which is independent of the parameter $\beta$, and therefore the variation $\delta S$ vanishes identically. The situation becomes even more drastically if one considers the Kerr solution. Being Ricci-flat, it still satisfies the field equations of Weyl conformal gravity. Furthermore it is asymptotically flat, so the definitions of mass and angular momentum given in [12 are applicable. As $R_{\mu \nu}=0=R_{\nu}^{\nu}, Y^{\mu \nu \rho \lambda}$ is zero in this case. The Noether current is proportional to $Y$ [20], hence it also vanishes. This implies that the entropy, mass and angular momentum of the Kerr black hole in Weyl conformal gravity are all zero, which means that the first law is satisfied in a trivial way. The vanishing of all these quantities certainly appears to be puzzling, and is closely related to the absence of an Einstein-Hilbert term and a fundamental length scale in the action (11). However, in [10] it was shown how one can still obtain the Bekenstein-Hawking entropy for the Schwarzschild black hole (at least at leading order) in Weyl gravity. One considers the breaking at quantum level of conformal invariance, generating thereby an effective Einstein-Hilbert action (see also [23]). In this approach the gravitational constant arises as an induced quantity in the spirit of Sakharov [11]; it is caused by the quantum fluctuations of matter fields in the vacuum. Let us shortly review how this works. The mentioned quantum fields $\Phi$ propagating in an external gravitational background $g$ induce an effective (euclidean) action $I_{i n d}[g]$ according to

$$
\exp \left(-I_{\text {ind }}[g]\right)=\int \mathcal{D} \Phi \exp \left(-I_{m}[\Phi, g]\right),
$$

where $I_{m}[\Phi, g]$ is the action of the matter fields. The total partition function $Z_{t o t}$ reads

$$
Z_{\text {tot }}=\int \mathcal{D} g \exp \left(-I_{g}[g]-I_{\text {ind }}[g]\right),
$$

$I_{g}[g]$ being the Weyl gravitational action given by (1). One can now show 10,23 (see also [25 27]) that $I_{\text {ind }}[g]$ contains an Einstein-Hilbert term. In order to obtain the black hole entropy, one calculates (28) in the saddle point approximation. For the Schwarzschild black hole this yields the Bekenstein-Hawking entropy, resulting from the action (27), plus correction terms, coming from (11). The Bekenstein-Hawking entropy is pure entanglement in this approach, it is generated by matter states hidden by an event horizon. (Interesting results on black hole entropy in induced gravity can also be found in [24 27]). Note however 
that the topological black hole solutions found in section [III do not solve the field equations following from the Einstein-Hilbert action. Therefore a calculation of the entropy as was done above for the Schwarzschild case would not yield the Bekenstein-Hawking result. Yet, if the quantum fluctuations induce also an effective cosmological constant (it is shown in [25] how this could work), the class of Einstein spaces among the topological black holes considered in the present paper are still solutions of the field equations following from the induced action. So one would obtain an entropy which is one quarter of the event horizon area, this part being pure entanglement, plus correction terms. In any case, the induced gravity approach to the entropy of the new black hole solutions found above seems to be more promising than the Noether charge method, which fails here due to the absence of a length scale.

\section{SUMMARY AND DISCUSSION}

In this paper we derived exact solutions of Weyl conformal gravity representing topological black holes. We found a generalization of the topological black holes which occur in AdS gravity. For genus $g>1$ this generalization includes spacetimes which are not asymptotically AdS, are globally hyperbolic and have vanishing scalar curvature at infinity. Moreover topological black holes with $g>1$ can also occur for a positive cosmological constant. On the other hand, in the toroidal case, only solutions which are asymptotically AdS occur.

As far as thermodynamical quantities are concerned, it makes sense to assign a temperature $T=\kappa / 2 \pi$ to our black hole solutions, because the field equations do not enter the derivation of the Hawking effect. However, the idea of black hole entropy as a Noether charge pioneered by Wald, and applicable to an arbitrary theory of gravity with diffeomorphism-invariant Lagrangian, does not yield satisfactory results in the case of Weyl conformal gravity, because the first law seems to be satisfied in a trivial way. This suggests that entropy and mass of black holes in Weyl gravity should be treated in the sense of [10], i. e. one does not assign any entropy to the classical gravitational field; rather it is generated by quantum matter states hidden by the event horizon.

Last, it seems worth mentioning that besides the fact that Weyl conformal gravity like AdS gravity admits black holes with unusual topology, these two theories of gravitation possess another common quality, being the only types of gravity which have a consistent interaction with massless higher spin fields [28.

\section{ACKNOWLEDGEMENT}

This work has been supported by a research grant within the scope of the Common Special Academic Program III of the Federal Republic of Germany and its Federal States, mediated by the DAAD.

The author would like to thank L. Vanzo for helpful discussions. 


\section{REFERENCES}

[1] S. Åminneborg, I. Bengtsson, S. Holst and P. Peldán, Class. Quantum Grav. 13, 2707 (1996).

[2] R. B. Mann, Class. and Quantum Grav. 14, L109 (1997);

R. B. Mann, gr-qc/9709039 (1997);

R. B. Mann and W. L. Smith, Phys. Rev. D 56, 4942 (1997).

[3] J. P. S. Lemos, Class. Quantum Grav. 12, 1081 (1995);

J. P. S. Lemos, Phys. Lett. B 353, 46 (1996);

J. P. S. Lemos and V. T. Zanchin, Phys. Rev. D 54, 3840 (1996).

[4] R. G. Cai and Y. Z. Zhang, Phys. Rev. D 54, 4891 (1996).

[5] C. G. Huang and C. B. Liang, Phys. Lett. A 201, 27 (1995);

C. G. Huang, Acta Phys. Sin. 4, 617 (1996).

[6] L. Vanzo, Phys. Rev. D 56, 6475 (1997);

D. Klemm, V. Moretti and L. Vanzo, Phys. Rev. D 57, 6127 (1998).

[7] D. Klemm and L. Vanzo, gr-qc/9803061 (1998) (accepted for publication in Phys. Rev. D).

[8] S. W. Hawking, Comm. Math. Phys. 25, 152 (1972).

[9] A. Bonanno, gr-qc/9801077 (1998), to appear on the proceedings of the workshop on The Internal Structure of Black Holes and Spacetime Singularities, Haifa, Israel, June 29 - July 3, 1997.

[10] B. Hasslacher and E. Mottola, Phys. Lett. B 99, 221 (1981).

[11] A. D. Sakharov, Sov. Phys. Doklady 12, 1040 (1968).

[12] R. M. Wald, Phys. Rev. D 48, 3427 (1993).

[13] B. S. DeWitt, in Relativity, Groups and Topology, Gordon \& Breach, New York (1964).

[14] P. D. Mannheim and D. Kazanas, Astrophys. Journal 342, 635 (1989).

[15] P. D. Mannheim, gr-qc/9407010.

[16] N. L. Balazs and A. Voros, Phys. Rep. 143, 109 (1986).

[17] R. G. Cai, J. Y. Ji and K. S. Soh, gr-qc/9708063.

[18] S. W. Hawking, Commun. Math. Phys. 43, 199 (1975).

[19] V. Iyer and R. M. Wald, Phys. Rev. D 50, 846 (1994).

[20] T. Jacobson, G. Kang and R. C. Myers, Phys. Rev. D 49, 6587 (1994).

[21] T. Jacobson, G. Kang and R. C. Myers, gr-qc/9502009 (1995).

[22] J. Koga and K. Maeda, gr-qc/9803086 (1998).

[23] B. Hasslacher and E. Mottola, Phys. Lett. B 95, 237 (1980).

[24] T. Jacobson, gr-qc/9404039 (1994).

[25] V. P. Frolov, D. V. Fursaev and A. I. Zelnikov, Nucl. Phys. B 486, 339 (1997).

[26] V. P. Frolov and D. V. Fursaev, Phys. Rev. D 56, 2212 (1997).

[27] V. P. Frolov and D. V. Fursaev, hep-th/9705207 (1997).

[28] E. S. Fradkin and M. A. Vasiliev, Nucl. Phys. B 350, 274 (1991). 